\title{
The effects of abrupt onset of 2-D and 3-D distractors on prehension movements
}

\author{
UMBERTO CASTIELLO \\ University of London, London, England
}

\begin{abstract}
This article includes two experiments aimed at investigating how two-dimensional (2-D) and threedimensional (3-D) distractors affect the kinematics of prehension and aiming movements in order to understand the attentional processes involved in visuomotor control. In Experiment 1, subjects grasped large targets in the presence of both large and small 3-D distractors and their corresponding 2-D photographs. The distractors appeared for either $10 \mathrm{sec}$ or appeared simultaneously with the target presentation. It was found that reach and grasp kinematics were influenced primarily by the small, suddenly appearing 3-D distractors. In Experiment 2, the purpose was to examine the conclusion that competition between objects (target and distractor) is related to the behavioral goal of the task. Experiment 2 is a replication of Experiment 1, with the exception that pointing movements were made instead of grasping movements. Results show that both 3-D and 2-D distractors interfered with pointing kinematics, supporting the hypothesis that attentional mechanisms are related to the goal of the task.
\end{abstract}

The notion that attention can be captured by the onset of new perceptual objects has been well documented in a series of experiments based on visual search tasks (Gibson, 1996a, 1996b; Yantis \& Jonides, 1984, 1996). In particular, it has been demonstrated that visual onsets capture attention because they coincide with the appearance of a perceptual object and not because of the sudden increment in luminosity (Yantis \& Jonides, 1984). Yantis and Jonides (1984) suggest that when a new object appears in the visual field it is necessary to create a new perceptual representation, and attention is typically directed toward it (Jonides \& Yantis, 1988; Yantis \& Jonides, 1996). As such, the new object may act as a nonspecific attentional distractor. Remington, Johnston, and Yantis (1992) demonstrated that response time to a letter target appearing in one of four boxes was increased if the target was preceded by distinct abrupt-onset visual stimuli flashed briefly in another box, in all boxes, or at fixation. This suggests that attention is captured involuntarily by abrupt onset.

In the present study, attention is not directly manipulated; however, it is conceivable to infer that the abrupt onset of a visual stimulus produces attentional capture. Instead of using typical laboratory generated stimuli (e.g., colored bars, $X \mathrm{~s}$ and $O \mathrm{~s}$ ) that bear little resemblance to the scenic objects that draw attention during naturally occurring tasks, we use a natural reach-to-grasp task per-

This research was sponsored by the Australian Research Council. I thank T. Sanocki, H. A. Sedgwick, K. Shapiro, and an anonymous referee for valuable comments and suggestions for improving the quality of the article. Correspondence concerning this article should be addressed to U. Castiello, Department of Psychology, Royal Holloway, University of London, Egham, Surrey, TW20 0EX, England (e-mail: u.castiello@rhul.ac.uk). formed toward real three-dimensional(3-D) objects (Jeannerod, 1981) to investigate attentional capture by abrupt onsets. The hypothesis that the abrupt illumination of a new perceptual object captures attention and can influence a planned overt motor action is tested. The general idea is that attention can access several processing modes, therefore several types of representations may operate simultaneously. For example, representations associated with object-oriented actions have distinct properties that make them distinct from those of other representations like object identification (Goodale \& Milner, 1992). Consequently, it is hypothesized that not only a perceptual but also a motor representation for the "distracting" object is created, attention is directed toward it, and perceptuomotor aspects of a new "object file" are opened. The prediction is that, at this point, both target and distractor objects can trigger competing reach-to-grasp programs, and the attentionally modulated resolution of this is played out in the kinematics of the reach to the target (Bonfiglioli \& Castiello, 1998; Castiello, 1996; Chieffi, Gentilucci, Allport, Sasso, \& Rizzolatti, 1993; Howard \& Tipper, 1997; Jackson, Jackson, \& Rosicky, 1995; Tipper, Howard, \& Jackson, 1997).

The link between selective attentional mechanisms and the control of action has already been observed in an experiment that forced a degree of covert attention to the distractor objects (see Castiello, 1996; Experiments 5 and 6). Subjects reached to grasp a target while having to count the number of times that a distractor object was illuminated by a spotlight. Under these circumstances, the characteristics of the distractor influenced the kinematics of the reach-tograsp movement. Specifically, the amplitude of peak grip aperture-that is, the distance between the index finger and the thumb-was influenced by the lateral distractor object. The amplitude of peak grip aperture for a target 
cherry was greater when a distractor larger than the cherry was present than when no distractor was present. The distractor thus appeared to disturb the correct motor output.

Bonfiglioli and Castiello (1998) studied the role of covert attention in the manifestation of distractor interference effects. In one of their experiments the target (an apple) was flanked by a distractor (a raspberry). A change in illumination during the movement indicated that the raspberry was to be grasped instead of the apple. One finding by Bonfiglioli and Castiello was that interference effects in the grasp, but not reach parameters were observed only when the distractor was smaller than the target and could potentially become the target. Specifically, peak grip aperture and grip aperture opening velocity were both reduced. This implies that the intrinsic features of a distractor (e.g., size), and not merely the extrinsic feature of location (Tipper et al., 1997), may elicit competing responses and thus have a selective influence on kinematic parameterization. However the location account cannot be discarded if it is assumed that the distractor was coded on the basis of the spatial location occupied. This hypothesis would imply that a two-dimensional (2-D) photographic image of the same diameter as the 3-D distractor used in previous studies (Bonfiglioli \& Castiello, 1998; Castiello, 1996) would produce the same interference effects.

Two issues are at stake in the present study. The first issue addresses the question of whether distractors in the form of 2-D photographic images or 3-D objects are represented differently. Farah (1990) has reviewed literature from a number of investigators that have shown differences in the ability of patients with visual agnosia to identify real objects as opposed to line drawings. For example, Efron (1969) described the symptoms manifested by a patient (Mr. S.) who showed a profound deficit in object recognition. He was unable to identify or copy line drawings of common objects, but he was able to recognize real objects. More recently, Milner and Goodale (1995) described the case of Mrs. D.F. As with the patient of the previous study, she was able to identify real objects rather than line drawings. Milner and Goodale explained these findings by proposing that both patients used surface properties rather than outline properties to identify objects (Humphrey, Goodale, Jakobson, \& Servos, 1994). In particular, it was suggested that properties such as color and texture, rather than outline shape, were used for object identification (Humphrey et al., 1994). Thus, if a dissociation between 2-D and 3-D stimulus material has been revealed, it is conceivable that mechanisms of attention devoted to select relevant properties necessary for 2-D and 3-D coding may operate at different levels and for different purposes when an action such as reaching-to-grasp is involved. To investigate the issue of dimensionality, 2-D and 3-D stimulus material were compared. In particular, subjects reached for a target in the presence of a distractor that was a 3-D object either similar or different in size from the target or in the presence of a 2-D distractor that was the photographic print of the 3-D distractors. These conditions should determine whether participants' reach-to-grasp actions were differentially affected by static 3-D or 2-D distractors. If volumetric properties of the distractor are important for grasping, the 2-D distractor should not produce the same interference on the grasp response as did the 3-D distractor.

The second issue follows from the first and addresses whether the sudden presentation of an irrelevant flanker object will affect kinematics of the reach-to-grasp movement to a target depending on task demands. The abrupt onset should trigger an object file representation providing temporary object-related information. As an irrelevant flanker, there is some question as to the extent to which the information contained within the object file integrates with that contained in the representation for the target object and the weighting it might contribute to the target motor action. To investigate the issue of task demands, pointing and grasping movements will be compared. Recent evidence suggests that visuomotor control can be different for pointing and grasping movements showing differences at the preprogramming and kinematic levels (Carnahan, Goodale, \& Marteniuk, 1993; Robertson, Nico, \& Hood, 1995, 1997), with grasping requiring a greater on-line computation than pointing. In particular, subjects were required to grasp (Experiment 1) and point (Experiment 2) to a target in the presence of a 3-D object or a 2-D photographic image distractor. It was predicted that when grasping is the task, sharing similar graspable properties is what matters. Therefore, because a 2-D flat photographic image does not share these properties, the action in progress should not be affected. When pointing is the task, sharing graspable properties is less relevant, given that pointing can be performed either to a 2-D photographic image or to a 3-D object. In the latter case, a 2-D flat photographic print distractor should affect the action in progress. This would support the notion that, for both 2-D and 3-D distractor objects, the identification process is similar, but the action selection process is affected with respect to the behavioral goal (i.e., the possible actions that the object affords).

The results reported here demonstrate that abruptonset objects interfere with grasping and pointing movements. When the task was to grasp a 3-D target object, the 3-D distractor interfered with aspects of movement, but the 2-D distractor did not. The critical variables affected were those related to the grasp component-namely, the time of maximum grip aperture, the amplitude of maximum grip aperture, and the rate of finger aperture. When the task was pointing, both distractor types interfered. The critical variables affected were movement duration and deceleration time. Thus, kinematics were differentially affected for 2-D and 3-D distractors for the grasping but not for the pointing task. It is suggested that the interfering effects of abrupt onset stimuli are dependent on task demands.

\section{EXPERIMENT 1}

In Experiment 1, we investigated whether reaching-tograsp for a 3-D object is affected by the rapid onset of 
distractor objects. In one condition, the distractor was a 3 -D object either similar or different in size to the target. This condition determined whether participants' reachto-grasp actions were affected by static 3-D distractors. In a second condition, the distractor was the photographic print of the 3-D distractors used in the other condition. If volumetric properties of the distractor are important, the 2-D distractor should not produce the same interference on the grasp response as the 3-D distractor.

\section{Method}

\section{Subjects}

Twenty students (10 women and 10 men, aged 20-25 years) volunteered to participate. All were right-handed (Edinburgh Inventory; Oldfield, 1971), reported normal or corrected-to-normal vision, and were naive as to the purpose of the experiment. They attended one experimental session of $\sim 1$-h duration.

\section{Type of Stimuli}

Stimuli consisted of (1) a 3-D target object (a red plastic apple; diameter, $\sim 8 \mathrm{~cm}$ ) positioned at a $30-\mathrm{cm}$ distance from the hand start- ing position along the midsagittal plane, (2) a 3-D distractor object, which was a red plastic apple similar to the target object (large distractor; diameter, $\sim 8 \mathrm{~cm}$ ) or a plastic cherry (small distractor; diameter, $\sim 2 \mathrm{~cm}$ ), and (3) 2-D distractor objects, which were the colored photographic prints of the same fruits. The 2-D photographic images were maintained in the vertical position by means of a cardboard support attached to the back of the photographic print. The cardboard was of the same shape and size as the photographic prints. The cardboard was cut so that it was not visible outside the outline of the object. The diameters for the objects represented in the photographic prints were equal to the diameters of the 3-D distractor objects ( 2 and $8 \mathrm{~cm}$, respectively). The brightness for the 2-D and the 3-D distractors was similar. The 2-D and 3-D distractor objects were presented vertically on the tabletop at $30^{\circ}$ to the right or left of the target (see Figure 1). After the experimental sessions, the subjects were asked to report what features of the photographic prints they could see. All subjects recognized the object represented both in the $2-\mathrm{D}$ and the $3-\mathrm{D}$ version.

\section{Apparatus}

Reflective passive markers $(0.25-\mathrm{cm}$ diameter) were attached to the wrist, the index finger, and the thumb. Movements were recorded with an ELITE motion analysis system. This consisted of
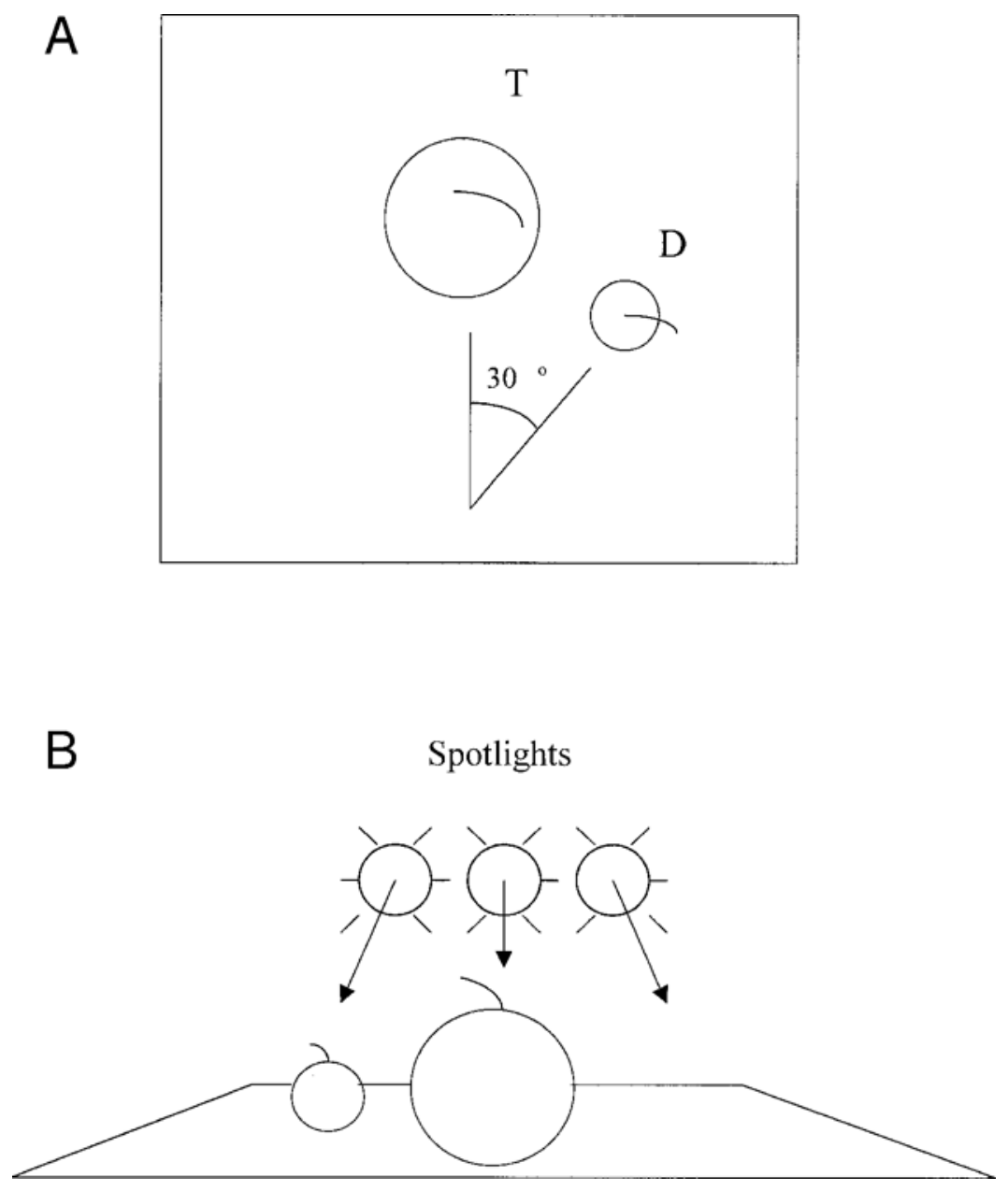

Figure 1. Experimental setup for Experiment 1. In panels A (top view) and B (front view) the target $(T=$ apple $)$ is represented with the small distractor $(D=$ cherry) on the right side. 
two infrared cameras (sampling rate, $100 \mathrm{~Hz}$ ) inclined at angles of $30^{\circ}$ to the vertical and placed $3 \mathrm{~m}$ in front of the table and $3 \mathrm{~m}$ apart. The spatial error measured from stationary and moving stimuli was $0.4 \mathrm{~mm}$. Coordinates of the markers were reconstructed with an accuracy of $1 / 3,000$ of the field of view and sent to a host computer.

Horizontal and vertical eye movements were recorded with an infrared corneal reflection system (sampling frequency, $120 \mathrm{~Hz}$ ). Trials in which eye movements in excess of $1^{\circ}$ visual angle (vertical and horizontal) were detected and replaced.

\section{Procedure}

The subject was seated at a table in a darkened room. The black working surface measured $90 \times 90 \mathrm{~cm}$ and was smooth and homogeneous. Prior to each trial, the right hand was placed on a pressuresensitive switch (diameter, $5 \mathrm{~cm}$ ) positioned $20 \mathrm{~cm}$ in front of the subject's midline. The illumination of the target and distractor indicated to the subjects that they should reach toward the target. The target and the distractor stayed on throughout the duration of the trial. Three spotlights were secured on stands with the hinges slightly inclined so that the 3-D objects or the photographic prints were illuminated from the front, one to the right, one to the center, and one to the left of the table (Figure 1). The spotlights were positioned behind and above the subject. The spotlight positioned to the right was calibrated to illuminate the distractor to the right. The spotlight positioned at the center was calibrated to illuminate the target. The spotlight positioned to the left was calibrated to illuminate the left distractor.

The subjects were required to fixate their eyes on the location where the target would appear throughout the trial. At the beginning of the experimental session and between each trial, the subjects were blindfolded by putting a mask in front of a glass frame where the infrared sensors were attached. During this period, either the 3-D distractor was changed or removed, or the 2-D photographic print was varied. In order to minimize the possibility that the subjects could see the type of distractor before its illumination, the delay between the removal of the mask and the illumination of the stimuli was minimal. It was not possible to control precisely for the delay between the removal of the mask and the illumination of the stimuli. An experimenter pressed the button to illuminate the stimuli as soon as she noticed through a mirror that the subject could see the working surface. A felt pad was attached to the bottom of the object to avoid unnecessary sound being an inadvertent cue for the subjects as to the type of distractor to be presented. Two control conditions were also included. For the first control condition (advance distractor), the target and the $3-\mathrm{D}$ distractor were visible $10 \mathrm{sec}$ before and throughout the duration of the entire trial. In this condition, the signal for the movement to begin was a computer generated tone $(880 \mathrm{~Hz}$; duration, $250 \mathrm{msec})$. Only the 3-D distractor in this condition was used. The rationale was that, at such a long exposure, the subjects would have perceived the 2-D picture as 3 -D given the volumetric properties of the cardboard support (2-mm width). In the second control condition (flash), only a flash of light similar to that used to present 3-D or 2-D distractors was presented until the trial ended, yet no object or photographic print was illuminated. In the final no distractor condition, no distractor appeared at all. The difference between the flash and the no distractor conditions is that only the spotlight for the target was activated.

The subjects performed 80 randomized trials over which all possible target/distractor combinations (10 trials for each combination) were presented. The main task of the subject was to reach and grasp the target at a leisurely pace.

\section{Data processing and analysis}

The ELIGRASP (B|T|S|, 1997) software package was used to analyze the data. This provided a 3-D reconstruction of the marker positions. The data were then filtered using a finite impulse response (FIR) linear filter-transition band of $1 \mathrm{~Hz}$ (sharpening variable $=2$; cut-off frequency $=10 \mathrm{~Hz}$ ). Movement initiation was taken from release of the starting switch. The end of the movement was taken as the time when the fingers closed on the target and there were no further changes in the distance between the index finger and thumb. No force sensors were used to detect the end of the start movement.

A common difficulty with kinematic studies concerns the number of dependent variables analyzed. Thus, the risk is of being unclear in regard to what scientific meaning a significant effect on some of these dependent variables would actually have. The logic that was applied here is in favor of a more economical approach. The dependent variables that were thought to be specifically relevant to the scientif ic hypothesis under test were specif ied and justified in advance, and the statistical analysis was confined to these variables.

Movement duration, the time between movement onset and the end of the action, was chosen because consistent results within the reach-to-grasp literature have been achieved for longer movement durations for smaller than for larger stimuli (Castiello, 1996; Gentilucci et al., 1991; Jakobson \& Goodale, 1992; Marteniuk, Leavitt, Mackenzie, \& Athenes, 1990). Thus, if a longer movement duration in the presence of a smaller distractor results, inferences regarding the influence of the small distractor on the kinematics of the larger target could be advanced (Bonfiglioli \& Castiello, 1998; Castiello, 1996; Kritikos, Bennett, Dunai, \& Castiello, 2000).

The reaching component was assessed by analyzing the velocity profile of the wrist marker, because there have been consistent results within the reach-to-grasp literature for the reaching component of a prolonged deceleration time (the time from peak velocity to the end of the movement) and a lower peak velocity amplitude for smaller than for larger stimuli (Castiello, 1996; Gentilucci et al., 1991; Jakobson \& Goodale, 1992; Marteniuk et al., 1990). Thus, if a longer deceleration time and/or a lower amplitude of peak velocity in the presence of a smaller distractor is found, inferences regarding the influence of the small distractor on the kinematics of the larger target could be advanced (Bonfiglioli \& Castiello, 1998; Castiello, 1996; Kritikos et al., 2000). The spatial trajectory of the arm was assessed because previous results have demonstrated differences in the lateral and vertical deviation of the spatial trajectory ( $x$ - and $z$-coordinates, respectively) as a function of the presence of distractors (Howard \& Tipper, 1997; Tipper et al., 1997).

The grasp component was assessed by analyzing the maximum distance between the two markers positioned on the index finger and thumb (amplitude of maximum grip aperture), the time when the maximum aperture occurred (time of maximum grip aperture), the amplitude of peak velocity of the fingers as they opened to a maximum grip (amplitude of peak grip velocity opening), and the time from maximum grip aperture to the close of the grip on the object (closing time). These dependent measures were chosen because consistent results within the reach-to-grasp literature is the anticipated and lowered amplitude of maximum grip aperture and a lower amplitude peak grip velocity for smaller stimuli than for large stimuli (Castiello, 1996; Gentilucci et al., 1991; Jakobson \& Goodale, 1992; Marteniuk et al., 1990). Thus, if the result of a lower and earlier occurrence of maximum grip aperture occurs, inferences regarding the influence of the smaller distractor on the grasp kinematics of the larger target can be postulated (Bonfiglioli \& Castiello, 1998; Castiello, 1996; Kritikos et al., 2000). The choice for analyzing the amplitude of peak grip velocity opening was determined by previous results in which the presence of the distractor influenced the rate of finger aperture (Bonfiglioli \& Castiello, 1998). The choice for analyzing closing time was motivated by the fact that it is a relevant index for the determination of planning reach-to-grasp strategies (Hoff \& Arbib, 1993).

For each dependent variable, two repeated-measures analyses of variance (ANOVA) were performed. In the first analysis, type of distractor (advance distractor, flash, or no distractor) was the withinsubjects factor. In the second analysis, type of distractor (advance distractor, 2-D or 3-D), distractor size (small or large), and distrac- 
tor position (right or left) were the within-subjects factors. The flash and the no distractor conditions were not included because, in these conditions, no distractor was present. Post hoc comparisons were conducted on the means of interest using the Newman-Keuls procedure $(\alpha=.05)$.

\section{Results}

In general, the results for the control conditions in which the distractor was visible before movement initiation (advanced distractor), when only the flash of light was presented (flash) and when the distractor was not present (no distractor), were very similar and did not reach statistical significance for any of the dependent measures (Table 1). Thus, for the sake of clarity, only the results from the second analysis will be reported. The advance distractor condition was chosen as a representative control condition because it was similar to the 2-D and the 3-D distractor conditions, except for the timing of the abrupt onset.

\section{Movement Duration}

For this parameter, the interaction of type of distractor $\times$ distractor size was significant $[F(1,19)=12.05$, $p<.001$; see Table 2]. Newman-Keuls comparisons revealed that movement duration was similar for the small and the large distractor for the advance distractor condition, whereas, for the 2-D and the 3-D distractor conditions, there were differences for movement duration with respect to distractor size (see Table 2). For the 2-D distractor condition, movement duration was longer for the large than for the small distractor $\left(p_{\mathrm{s}}<.05\right.$; Table 2$)$. For the 3-D distractor condition, movement duration was longer for the small than for the large distractor $\left(p_{\mathrm{s}}<.05\right.$; Table 2). Furthermore, movement duration for the large 3 -D distractor condition was similar to movement duration for the large 2-D distractors (Table 2). No effects for distractor position were found $[F(1,19)=0.45]$. Movement duration was similar when the distractor was positioned to the right or to the left of the target.

\section{Reaching Component}

Deceleration time. As revealed by the significant interaction between type of distractor and distractor size
$[F(1,19)=32.12, p<.0001$; see Table 2$]$, the time from peak velocity to the end of the movement varied significantly with respect to distractor size only for the 3-D distractor condition $\left(p_{\mathrm{s}}<.05\right.$; see Table 2$)$. Deceleration time was longer for the 3-D small than for the 3-D large distractor. The difference in deceleration time between the small and the large size distractor for the advanced and the 2-D distractor conditions was not significant. No significant effects of distractor position were found $[F(1,19)=1.44]$. Deceleration time showed similar values when the distractor was positioned to the right or to the left of the target $[F(1,19)=0.65]$.

Spatial trajectories. The analysis of wrist marker trajectories showed that there were no significant differences for the amplitude of maximum lateral and vertical deviations as calculated from the wrist marker between the different distractor conditions. This result is not in agreement with Tipper et al.'s study (1997; see also Howard \& Tipper, 1997), in which there were interference effects on movement trajectory. However, there are many critical differences between this study and that of Tipper et al. (1997). For example, in the Tipper et al. (1997) study the target and distractor could be in one of four possible loci, and this was not known in advance. In sharp contrast, in the present study, the target location was known in advance and was constant throughout the experiment, enabling substantial preplanning. Indeed, when target and distractor loci were more predictable, Tipper et al. (1997) also failed to observe trajectories effects.

Amplitude of maximum peak velocity. The amplitude of maximum peak velocity did not differ with respect to type of distractor $[F(1,19)=1.32]$ and distractor size $[F(1,19)=0.87]$. The interaction between type of distractor and distractor size was not significant $[F(1,19)=$ 1.02].

\section{Grasp Component}

Amplitude of maximum grip aperture. The results obtained for the maximum opening of the hand suggest interference effects on movement parameterization. The significant interaction between type of distractor and distractor size $[F(1,19)=31.04, p<.0001]$ revealed that, when the 3-D distractor was smaller than the target, the

Table 1

Movement Duration and Kinematic Parameters Values for the Three Control Conditions for Experiment 1

\begin{tabular}{|c|c|c|c|c|c|c|}
\hline \multirow[b]{2}{*}{ Variable } & \multicolumn{2}{|c|}{$\begin{array}{l}\text { Advanced } \\
\text { Distractor }\end{array}$} & \multicolumn{2}{|c|}{ Flash } & \multicolumn{2}{|c|}{$\begin{array}{c}\text { No } \\
\text { Distractor }\end{array}$} \\
\hline & $M$ & $S E$ & $M$ & $S E$ & $M$ & $S E$ \\
\hline Movement duration (msec) & 781 & 19 & 779 & 18 & 784 & 18 \\
\hline \multicolumn{7}{|l|}{ Reaching component } \\
\hline Deceleration time (msec) & 468 & 11 & 479 & 12 & 467 & 12 \\
\hline Amplitude peak velocity $(\mathrm{mm} / \mathrm{sec})$ & 449 & 12 & 449 & 13 & 450 & 11 \\
\hline \multicolumn{7}{|l|}{ Grasp component } \\
\hline Time of maximum grip aperture (msec) & 542 & 13 & 538 & 12 & 545 & 13 \\
\hline $\begin{array}{l}\text { Amplitude of peak grip velocity } \\
\text { opening }(\mathrm{mm} / \mathrm{sec})\end{array}$ & 449 & 12 & 447 & 12 & 456 & 11 \\
\hline $\begin{array}{l}\text { Amplitude of maximum grip } \\
\text { aperture (mm) }\end{array}$ & 106 & 1 & 106 & 1 & 110 & 1 \\
\hline Closing time (msec) & 239 & 7 & 241 & 6 & 239 & 5 \\
\hline
\end{tabular}


Table 2

Movement Duration and Kinematic Parameter Values for the Interaction Between Type of Distractor and Distractor Size for Experiment 1

\begin{tabular}{|c|c|c|c|c|c|c|c|c|c|c|c|c|}
\hline \multirow[b]{3}{*}{ Variable } & \multicolumn{4}{|c|}{ Advanced Distractor } & \multicolumn{4}{|c|}{ 2-D } & \multicolumn{4}{|c|}{$3-\mathrm{D}$} \\
\hline & \multicolumn{2}{|c|}{ Small } & \multicolumn{2}{|c|}{ Large } & \multicolumn{2}{|c|}{ Small } & \multicolumn{2}{|c|}{ Large } & \multicolumn{2}{|c|}{ Small } & \multicolumn{2}{|c|}{ Large } \\
\hline & $M$ & $S E$ & $M$ & $S E$ & $M$ & $S E$ & $M$ & $S E$ & $M$ & $S E$ & $M$ & $S E$ \\
\hline Movement duration (msec) & 769 & 20 & 773 & 19 & 794 & 20 & 816 & 19 & 840 & 24 & 818 & 24 \\
\hline \multicolumn{13}{|l|}{ Reaching component } \\
\hline Deceleration time (msec) & 469 & 12 & 493 & 14 & 500 & 13 & 489 & 12 & 583 & 14 & 544 & 15 \\
\hline Amplitude peak velocity $(\mathrm{mm} / \mathrm{sec})$ & 445 & 13 & 439 & 13 & 457 & 13 & 487 & 12 & 439 & 11 & 452 & 11 \\
\hline \multicolumn{13}{|l|}{ Grasp component } \\
\hline Time of maximum grip aperture (msec) & 527 & 15 & 535 & 13 & 545 & 14 & 558 & 13 & 467 & 17 & 543 & 13 \\
\hline $\begin{array}{l}\text { Amplitude of grip peak velocity } \\
\text { opening }(\mathrm{mm} / \mathrm{sec})\end{array}$ & 462 & 11 & 471 & 12 & 462 & 11 & 444 & 11 & 499 & 15 & 455 & 12 \\
\hline $\begin{array}{l}\text { Amplitude of maximum grip } \\
\text { aperture (mm) }\end{array}$ & 105 & 1 & 107 & 1 & 107 & 1 & 105 & 1 & 86 & 1 & 102 & 1 \\
\hline Closing time (msec) & 242 & 7 & 238 & 6 & 249 & 6 & 258 & 6 & 373 & 9 & 275 & 7 \\
\hline
\end{tabular}

hand opened less than when the target was grasped in the presence of the 2-D or the advanced distractor (NewmanKeuls, $p_{\mathrm{s}}<.05$; see Table 2). For the two latter conditions, the amplitude of maximum grip aperture was similar with respect to the size of the distractor. No significant effects of distractor position were found. The amplitude of maximum grip aperture was not influenced by the position of the distractor $[F(1,19)=2.65]$. It was the same whether the distractor was positioned to the right or to the left of the target.

Time of maximum grip aperture. For this parameter, the interaction of type of distractor $\times$ distractor size was significant $[F(1,19)=12.11, p<.001]$. When the $3-\mathrm{D}$ distractor was smaller than the target, the hand reached the maximum grip aperture (i.e., the maximum distance between the index finger and thumb) earlier than when the target was grasped in the presence of the large 3-D distractor (Newman-Keuls, $p_{\mathrm{s}}<.05$; see Table 2 ). In contrast, in the advanced and 2-D distractor conditions, maximum grip aperture was reached at similar times for the large and the small distractors (see Table 2). This result suggests interference effects on movement parameterization. No differences were found with respect to distractor position $[F(1,19)=0.98]$. The distractor thus appeared to disturb the correct output independently from its position.

Closing time. The interaction of type $\times$ distractor by distractor size was significant $[F(1,19)=14.21, p<$ $.0001]$. When the 3 -D distractor was smaller than the target, the time from the maximum grip aperture to the end of the movement was longer than when the target was grasped in the presence of the 3-D large distractor (Newman-Keuls, $p_{\mathrm{s}}<.05$; see Table 2). For the 2-D and the advanced distractor conditions, no effects of size were found. The position of the distractor did not influence closing time $[F(1,19)=1.87]$.

Amplitude of peak grip velocity opening. As revealed by the significant interaction between type of distractor and distractor size $[F(3,57)=54.67, p<.0001]$, when the 3-D distractor was smaller than the target, the hand opened at a faster rate than when the target was grasped in the presence of the 3-D large distractor (NewmanKeuls, $p<.05$; see Table 2). For the 2-D and the advanced distractor conditions, no differences with respect to distractor size were found. No significant effects due to the position of the distractor were found $[F(1,19)=0.65]$.

\section{Discussion}

In the present experiment, the subjects grasped large targets with the presence of large or small 3-D distractors before target presentation or their corresponding 2-D photographs. The distractor appeared either $10 \mathrm{sec}$ before target presentation, or simultaneously with the target presentation. It was found that reach and grasp kinematics were influenced primarily by the 3-D suddenly appearing distractors.

Except for the magnitude of peak velocity, all other dependent measures showed significant changes in the direction of the experimental hypothesis. That is, the classic kinematic patterning that characterizes the reach-tograsp for large objects (target) was modified according to the classic kinematic pattern that characterizes smaller objects (small distractor; Gentilucci et al., 1991; Marteniuk et al., 1990). Movement duration and deceleration time was prolonged, and the time of maximum grip aperture was anticipated.

A possible explanation for the interference effects found when the 3-D large distractor was presented with the 3-D large target is that, apart from the size dimension, "graspability" is a fundamental issue. Response assignment within the task dimension might be linked to a central response selection process for motor output formation. For example, competition may arise, because the target and the distractor objects are associated on the basis of the same size and type of response codes. They activate competing responses within the grasp task and the size modules. Competition might arise because the target and the distractor objects are associated on the basis of the 
same size code. In other words, the response to the target is affected by the distractor not necessarily when they have different characteristics or require different responses but even when both are similar (Cohen \& Shoup, 1997). The features from the distractor and the target objects that belong to a particular attribute are analyzed simultaneously, producing a congruency effect that determines interference.

The basic effect obtained in this experiment has implications for models of response selection. Multiple attended objects compete for action, each perhaps generating parallel kinematic plans for grasping. As such, irrelevant objects can produce interference effects that can be distinct for 2-D and 3-D distractors. The interference effects created by a distractor object are mainly restricted to the 3 -D condition. In particular, only 3-D distractor objects produce activation of grasping representations. Thus, a dissociation in which interference from 3-D objects is greater on measures related to manipulation was found. As an example, the subjects in the present study always reached for the same target object, an apple of $80 \mathrm{~mm}$ in diameter, producing mean maximum grip apertures that ranged from 102 to $107 \mathrm{~mm}$. This indicates a safety margin of around $30 \%$ - a result that is in line with the previous literature on prehension movements. However, only in the 3-D/small distractor condition, was the mean maximum grip aperture $86 \mathrm{~mm}$. This was only $6 \mathrm{~mm}$ greater than the size of the target object and represents a safety margin of only $7.5 \%$. This striking reduction in the safety margin may thus be interpreted as a confirmation of the strong level of interference produced by the $3-\mathrm{D} / \mathrm{small}$ distractor.

At first glance, it would seem that the present results provide clear answers to the questions addressed in this study: (1) Differential interference effects are found for 2-D and 3-D distractors. (2) Interference effects appear to be task related. When grasping is the task, it is the volumetric properties of the object that matters. (3) Interference effects emerge only when the distractor attracts attention. However, it would be an overstatement to argue that interference effects only arise from 3-D objects, depending on the type of task without having tested other actions that the distractor objects could afford. It would be expected that the system is more loosely organized. That is, all irrelevant objects can produce interference effects, though the weight attached to them will vary as a function of task. The 2-D shapes that were chosen were not graspable but, for example, they may have been targets for a pointing action. If interference is task related, asking the subjects to point toward the 3-D target in the presence of the 2-D distractor should reveal interference. To explore for this hypothesis the issue of task demand is addressed in Experiment 2.

\section{EXPERIMENT 2}

Results from the previous experiment suggest the notion that competition between objects is also related to the behavioral goal of the task. In particular, in order to reveal interference effects at a motor level, specific properties of the target and distractor objects must be associated with action systems. At this stage, however, the task relevant hypothesis rests on a null effect. That is, the 2-D projected shape distractors did not affect the grasp component. It is thus necessary to obtain evidence that shows that, when 2-D shapes are task-relevant, interference effects are also produced. One way to do this is to produce a variation in the task that would potentially elicit interference from the 2-D distractors. To do this, the subjects were asked to perform a pointing task compatible with both the 3-D target and the 2-D projected shapes. A projected shape cannot be grasped, yet it is possible to point to it.

Recent studies have suggested that visuomotor control for pointing and grasping can be different. For example, differences have been found between pointing and grasping in neglect patients (Robertson et al., 1995; see also Robertson et al., 1997). In these studies, patients were required either to point to or grasp a rod positioned to the right, centrally, or to the left. A strong rightward bias in their performance was evident only with the pointing action. Robertson et al. $(1995,1997)$ suggested that the frame of reference used to represent visual information is different for pointing and grasping. Furthermore, they suggest that, given the preplanning differences for pointing and grasping (see Carnahan et al., 1993), the attentional demands for grasping may be different than those for pointing (Robertson et al., 1995, 1997).

\section{Method}

\section{Subjects}

Twenty students ( 10 women and 10 men, aged $22-26$ years) with the same characteristics as those that took part in the previous experiments volunteered to participate; none had participated in the previous experiment. All were naive as to the purpose of the experiment and attended one experimental session of $.5 \mathrm{~h}$ duration.

\section{Stimuli and Apparatus}

The same apparatus and stimuli as for Experiment 1 were used.

\section{Procedure}

The procedure was the same as that of Experiment 1, with the following exception: Subjects were required to point to the target instead of grasping it.

\section{Data Analysis}

Data were analyzed as for the previous experiment. Given the nature of the task, only the dependent measures relative to the reach component were analyzed (i.e., movement duration, deceleration time, and the amplitude of peak velocity). As for the previous experiment, no differences were found between the advanced, flash, and the no distractor conditions. Thus, only the advanced control condition is reported as a mean of comparison with the 2-D and 3-D distractor conditions.

\section{Results}

The main factor of type of distractor was significant for movement duration $[F(1,19)=16.13, p<.0001]$ and for deceleration time $[F(1,19)=14.12, p<.001]$. Post hoc contrasts revealed that movement duration and the time 

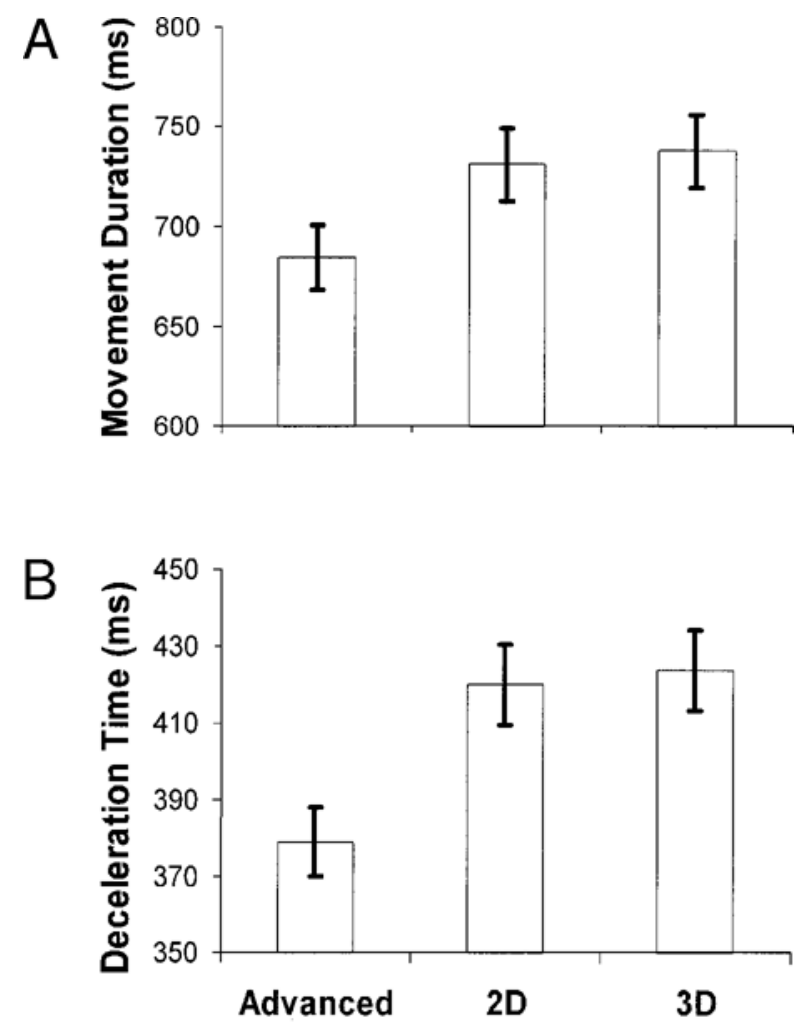

\section{Type of Distractor}

Figure 2. Graphic representation of the main factor type of distractor for movement duration (panel $A$ ) and deceleration time (panel B) for Experiment 2.

spent in decelerating were greater for the 2-D and the 3-D than for the advance distractor conditions (movement duration, 731, 738, and $685 \mathrm{msec}$, respectively; deceleration time, 420, 424, and $379 \mathrm{msec}$, respectively; see Figure 2). The difference in deceleration time between the small and the large distractor for the advanced, the 2-D, and the 3-D distractor conditions was not significant. However, although this difference did not reach significance, deceleration time was longer for the small than for the large distractor for both the 2-D and the 3-D conditions $(2-\mathrm{D}=428$ vs. $412 \mathrm{msec} ; 3-\mathrm{D}=431$ vs. $417 \mathrm{msec})$. No significant differences for distractor position were found.

\section{Comparison Analyses}

In order to investigate whether distractors influenced grasping and pointing differentially, three ANOVAs, one for movement duration, one for deceleration time, and one for the amplitude of peak velocity, were conducted. The between-subjects factor was type of reaching (Reaching Experiment 1 vs. Pointing Experiment 2) and the withinsubjects factor was type of distractor (2-D, 3-D, or advance distractor). For deceleration time and movement duration, the interaction between type of reaching and type of dis- tractor was significant for movement duration $[F(1,19)=$ $5.01, p<.05]$ and deceleration time $[F(1,19)=4.54, p<$ $.05]$. Movement duration and deceleration time were longer for the 3-D than for the 2-D distractor condition only for the grasping action (pointing: $424 \mathrm{vs} .420 \mathrm{msec}$, respectively; grasping: 564 vs. $494 \mathrm{msec}$, respectively; see Figure 3). Deceleration time was also analyzed in relative terms, as a percentage of movement duration, and led
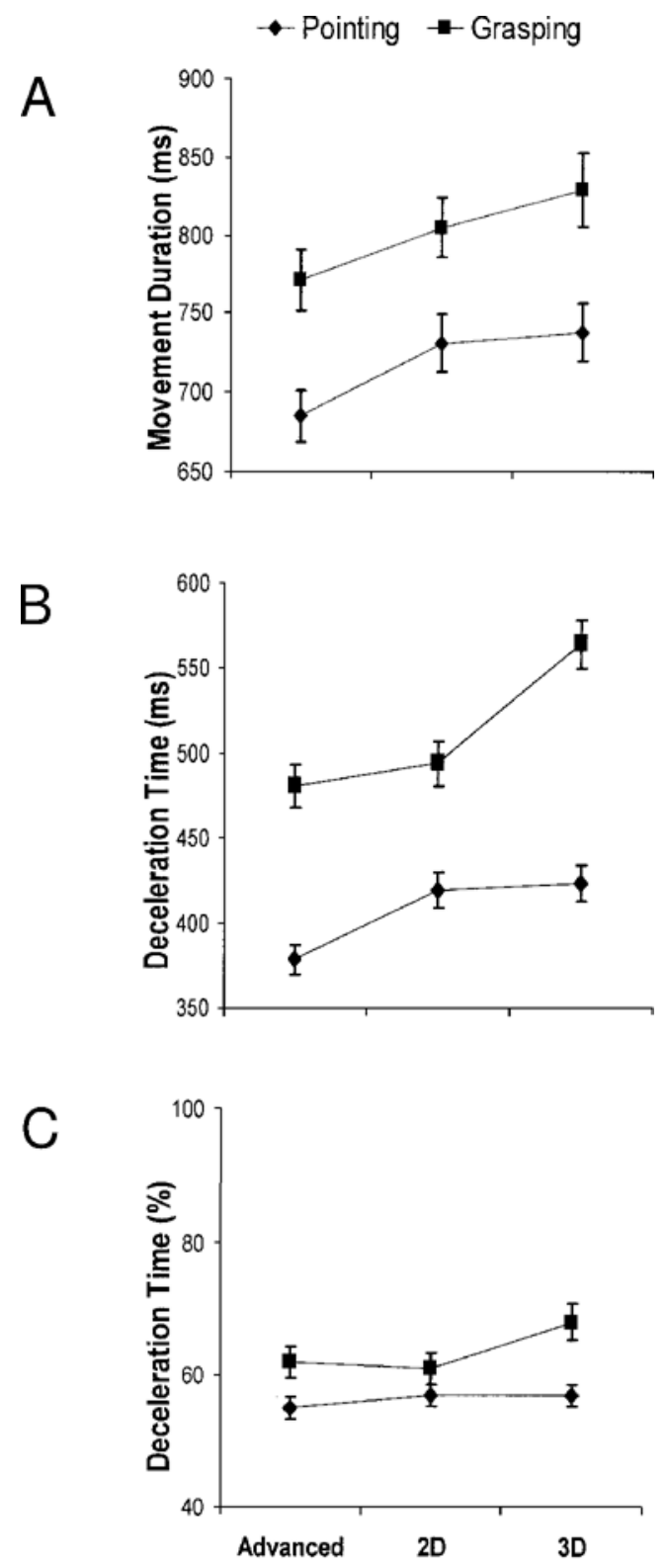

\section{Type of Distractor}

Figure 3. Graphic representation of the interaction between type of reaching (pointing vs. grasping) and type of distractor (advanced, 2-D, and 3-D) for movement duration (panel A), deceleration time in absolute values (panel $B$ ), and deceleration time in relative terms (\%, panel C) for Experiment 2. 
to similar results. The interaction between type of reaching and type of distractor was significant $[F(1,19)=$ $10.43, p=.001$; see Figure 3].

\section{Discussion}

These results provide evidence that attentional mechanisms are flexible and influenced by the goal of the task (Tipper, Weaver, \& Houghton, 1994). Attention selects not only the object in its entirety, but also specific properties that are task-relevant. Sources of conflict or competition depend on the particular processing systems that tasks require in common. In the present context, competition might be sensitive to the attributes of irrelevant objects that match those of the object relevant to the end goal. In the present experiment, the 3-D distractors required a similar type of pointing action as the target object. The parallel computation for different pointing actions, one for the target and one for the attended distractor, might be the origin of the changes noticed for the kinematics of the action directed to the target.

The differences found for pointing and grasping in the present experiment seem to confirm previous observations of visual neglect studies, suggesting a differential visual coding according to the type of motor action performed by subjects (Edwards \& Humphreys, 1999; Robertson et al., 1995, 1997). It was found that picking up a metal rod produces less neglect in the form of a smaller deviation to the person's right side than when pointing to the perceived center of the same rod. These results suggest how visual information is selectively available, depending on the type of motor responses to an object. In attentional terms, this formulation points to a multiplicity of spatial attentional mechanisms that are task dependent and not only associated with perceptual analysis of objects. This view seems confirmed by the notion proposed by Milner and Goodale (1995) that mechanisms of attention operate in concert at both ventral and dorsal stream levels to subserve actions directed to objects. These authors hypothesized that the ventral stream carries out the first selection process for target and distractor objects where the goal and the task directed to objects is determined. This coding is transferred to the dorsal stream, which facilitates actions directed to the objects. If this is how the system works, the interference effects found in the present experiment can be "dorsal" in nature. If the ventral system sends information for both the target and the distractor to the dorsal system, and both objects afford the same action, activity within the dorsal stream is intensified so that the two objects compete at motor output level.

Possible alternative explanations for the difference between grasping and pointing are related to processing time. In general, grasping responses are much longer overall than pointing responses. A possible reason is that a percept of a 3-D object develops over time. For example, as demonstrated by Sekuler and Palmer (1992) object processing begins with a 2-D retinal mosaic and develops into a 3-D percept. If so, the $3-D$ percept is likely to be more developed in the long-response grasping ex- periment than in the quick-response pointing experiment. Thus, 3-D versus 2-D differences would likely be stronger in the grasping experiment, for perceptual reasons. However, by looking at the data for deceleration time as a percentage of movement duration, the differential pattern for grasping and pointing is still present. Because the percentage of time spent decelerating differs with respect to distractor condition also in relative terms (\%), this signifies that it is the type of distractor and not the duration of the action that determines the difference. In other words, it is not because movement duration is longer for grasping that the 3-D versus 2-D effect is stronger or because pointing actions are faster that the 3-D versus 2-D effect is not evident. If this were the case, in relative terms, deceleration time for the grasping condition might not be, for example, different for 2-D and 3-D distractor conditions or, as another example, the 2-D and 3-D distractor condition may differ for pointing.

An effect of size was also expected, given the differences found for pointing movements to small and large targets (Gentilucci et al., 1991; Mackenzie, Marteniuk, Dugas, Liske, \& Eickmeier, 1987; Marteniuk et al., 1990). However, although there is a trend in this direction, no significant differences were found for any of the dependent measures for the main factor of size and the interaction between type of distractor and distractor size. This discrepancy is probably due to the fact that the stimuli used in the present experiment did not vary in size so as to determine a differential level of accuracy for a pointing task (Marteniuk, MacKenzie, Jeannerod, Athenes, \& Dugas, 1987).

\section{GENERAL DISCUSSION}

The central question explored in this investigation was whether 2-D and 3-D object information presented suddenly is processed differently by the visuomotor system. In the first experiment, it was found that the abrupt onset of a distractor object interfered with the duration and the kinematics of the reach-to-grasp movement toward the target object. However, when subjects could view the distractor object before movement initiation, no interference effects were observed. Furthermore, the distracting effects of stimuli were evident when they consisted of 3 -D objects compared with when they were 2-D prints of equivalent size. In Experiment 2, the idea of a task relevant hypothesis in which objects compete on the basis of the task they afford appeared to be confirmed. When pointing is the task, the $2-\mathrm{D}$ photographic print did produce interference.

The reach-to-grasp action is a goal-directed action that requires some degree of attention to the stimulus to be grasped. This movement is performed regularly in the presence of task-irrelevant objects, and there is much debate as to whether the presence of flanking objects influences organization of the movement to the target (for a review, see Castiello, 1999). The degree to which attention is drawn toward the distracting object is one factor that might determine the strength of a distracting/facilitating effect of a flanker object on the target action. 
If results from the computer screen environment can be extrapolated to a 3-D world, it can be proposed that abrupt onset of a flanker object might act to capture attention in a more automatic manner than the voluntary deployment strategy used in previous studies (Castiello, 1996). However, because action is goal directed toward the target, voluntary intent might override any involuntary attraction to the flanker. Successful overriding by voluntary intent would be indicated by the lack of interference effects under conditions of abrupt flanker onset. In the present experiments, voluntary intent, or at least task, did moderate interfering effects coming from the flanker object. The appearance of a new perceptual object might trigger the creation of a new object representation, and this in turn attracts attention. The result of this attentional orienting is the automatic activation of motor responses to the distractor, which was never overtly reached throughout the study. The simultaneous activation of prehension to both a target and distractor produces cross-talk in the neural population codes representing them, hence the properties of the grasp evoked by the distractor contaminate those evoked by the target (e.g., Tipper, Howard, \& Houghton, 2000).

These results thus suggest implicit processing of the distractor from a stimulus driven perspective but also enlarge this notion to an action representation point of view. That is, the type of representation created for the distractor contains information about the action that the object affords, and this action, if incompatible, competes with the action programmed for the target object.

A number of experiments have demonstrated that interference occurs when features of different objects must be processed simultaneously (Vecera \& Farah, 1994). The present results suggest that the special attentional status attributed to new objects might also be derived by their motor quality features. When the distractor is presented, a goal-based search process might start. This process involves the scanning of the motor schema storage, which better adapts to that specific object. The consequence is the implicit selection of the action structure that best satisfies structural requirements. If the retrieved motor schema for the distractor object clashes with the target motor schema, interference emerges.

Suggestions that the results of the present study demonstrate a conflict between the task attributes of the distractor and those of the target come from the finding that, when the distractor is a 3-D object, both the kinematics of the transport and the manipulation components are influenced. When the distractor is a 2-D shape, kinematic conflict is not evident. Although a trend in the data for some dependent measures suggested that it may also be possible to elicit kinematic interference from a 2-D projected shape, the interesting point for discussion is that stronger interference emerges at the grasping level when the distractor is a 3-D object. The 3-D object distractor competed in terms of the grasping action required and in terms of its volumetric properties. For example, when the 3-D distractor was smaller than the target, the hand opened less and faster than when the target was grasped in isolation. When the distractor was a 3-D object similar to the target, the speed of finger aperture was affected but not the size of the aperture. When the distractor was a 2-D projected shape, these effects were not present.

The small and the large 3-D distractor objects utilized in the present study required different types of prehension than did the target object (i.e., precision grip, whole hand prehension). Thus, when the distractor is small, parallel computations for different types of grasp, one for the target and one for the attended distractor, may be the origin of the changes found for the kinematics of the action directed to the target. This view is supported by neurophysiological and behavioral evidence. In the first case, different types of grasp or given types of action are subserved by different neural populations (Rizzolatti et al., 1988; Sakata \& Taira, 1994). In the second case, the kinematics differ for different types of grasp. For instance, the time course of the manipulation component and its temporal relations with the transport component change with the type of grasp (Castiello, 1996; Gentilucci et al., 1991).

Further evidence from Klatzky, McCloskey, Doherty, Pellegrino, and Smith (1987) demonstrates that knowledge about the object specifies the pattern of hand contact. They distinguished between hand-shape representations associated with objects in memory and showed how such representations are related to the structural and functional properties of objects. In light of this evidence, it can be suggested that conflicts can emerge when the distractor and target objects require different prehensile patterns in order to be grasped or manipulated. Neuronal populations, kinematic planning, and functional properties for the irrelevant distractor object are alerted and interfere with neuronal populations, kinematic planning, and functional properties activated and executed for the target object. In other words, distractors automatically activate their responses without the subject's intention to act (e.g., Lhermitte, 1983). Given this automatic process of conversion of perceptual input into the action afforded by the distractor objects, different objects in a visual scene can evoke the parallel implementation of actions (Eriksen, 1995; Goldberg \& Segraves, 1987; Tipper et al., 1997). If more than one motor pattern is kept active at a time, this parallel activation determines mutual interference.

A task-relevant hypothesis is proposed; distractors can be expected to interfere because they share specific task relevant properties. When grasping is the task, sharing different or similar graspable properties is what matters; 2-D shapes projected on the table top do not share this. However, when both the 3-D target and the 2-D distractor are common to the task, interference emerges. This is clearly demonstrated in Experiment 2 in which pointing was the task. Pointing tasks can be performed both on a 3 -D object and a 2-D projected shape. However, an alternative hypothesis might be that the abrupt appearance of the 3-D distractor not only enhances grasping prepara- 
tion for the distractor, but also activates some preparation to point at the distractor. In other words, is preparation to point at the distractor only activated when the subject is preparing to point at the target or also when the subject is preparing to grasp the target? ${ }^{1}$ Although, all the data in the present study would appear to be consistent with the view that only the task that is about to be performed on the target can be evoked by the distractor, the latter possibility deserves further investigation.

With a certain degree of caution, it could be advanced that the present findings provide an indication that the abrupt onset of a distractor object captures attention in a stimulus-driven fashion. However, because the appearance of both the target and the distractor were defined by abrupt onset, one can reasonably assert that the subjects were actively looking for the abrupt appearance of the target stimulus on each trial. Consequently, abrupt onset was a highly relevant feature in the present study. Therefore, no strong conclusions can be drawn about the nature of attentional control.

A final point is concerned with the fact that the distractors were viewed only through peripheral vision (since the eyes always fixated the target). This may allow speculations regarding the implications of peripheral visual input into the discrimination of 2-D versus 3-D target. Sivak and MacKenzie (1990) have examined the contribution made by peripheral and central vision to reaching and grasping. They found that, with only peripheral vision, information related to the size of an object was inadequate to unfold the grasp component properly. If the volumetric intrinsic properties of an object are poorly coded with peripheral vision (covert attention), this would suggest that the attentional coding for the 3-D distractor object is more demanding than that for the 2-D shape, which does not present volumetric properties. Again, this points to a spatial attentional system that is able to intensify the visual processing of particular object features to achieve different ends.

In conclusion, this study identifies new conditions determining the disruption of kinematics for normal selective movements in response to irrelevant information. The identification of these factors is a critical step in the development of useful measures for studying selection for action, object representation, and selective attentional mechanisms. Whether or not visual information can be attended to simultaneously is not only contingent on information load but it also depends on functional and structural factors of the stimulus, such as depth structure.

\section{REFERENCES}

Bonfiglioli, C., \& Castiello, U. (1998). Dissociation of covert and overt spatial attention during prehension movements: Selective interference effects. Perception \& Psychophysics, 60, 1426-1440.

Carnahan, H., Goodale, M., \& Marteniuk, R. (1993). Grasping versus pointing and the differential use of visual feedback. Human Movement Science, 12, 219-234.

CAstiello, U. (1996). Grasping a fruit: Selection for action. Journal of Experimental Psychology: Human Perception \& Performance, 22, 582-603.
Castiello, U. (1999). Mechanisms of selection for the control of hand action. Trends in Cognitive Sciences, 7, 264-271.

Chieffi, S., Gentilucci, M., Allport, A., Sasso, E., \& Rizzolatti, G. (1993). Study of selective reaching and grasping in a patient with unilateral parietal lesion. Brain, 116, 1119-1137.

Cohen, A., \& Shoup, R. (1997). Perceptual dimensional constraints in response selection processes. Cognitive Psychology, 32, 128-181.

Edwards, M., \& Humphreys, G. (1999). Pointing and grasping in unilateral visual neglect: Effect of on-line visual feedback in grasping. Neuropsychologia, 37, 959-973.

EFron, R. (1969). What is perception? Boston Studies in the Philosophy of Science, 4, 137-173.

ERIKSEN, C. (1995). The flankers task and response competition: A useful tool for investigation of a variety of cognitive problems. Visual Cognition, 2, 101-118.

FARAH, M. (1990). Visual agnosia: Disorders of object recognition and what they tell us about normal vision. Cambridge, MA: MIT Press.

Gentilucci, M., Castiello, U., Corradini, M. L., Scarpa, M., Umiltà, C., \& Rizzolatti, G. (1991). Influence of different types of grasping on the transport component of prehension movement. Neuropsychologia, 29, 361-378.

GiBSon, B. (1996a). The masking account of attentional capture: A reply to Yantis and Jonides. Journal of Experimental Psychology: Human Perception \& Performance, 22, 1514-1520.

GiBson, B. (1996b). Visual quality and attentional capture: A challenge to the special role of abrupt onsets. Journal of Experimental Psychology: Human Perception \& Performance, 22, 1496-1504.

Goldberg, M. E., \& Segraves, M. A. (1987). Visuospatial and motor attention in the monkey. Neuropsychologia, 25, 107-118.

Goodale, M. A., \& Milner, A. D. (1992). Separate visual pathways for perception and action. Trends in Neurosciences, 15, 20-25.

Hoff, B., \& ARBIB, M. A. (1993). Models of trajectory formation and temporal interaction of reach to grasp. Journal of Motor Behavior, 25, 175-192.

Howard, L. A., \& Tipper, S. P. (1997). Hand deviations away from visual cues: Indirect evidence for inhibition. Experimental Brain Research, 113, 144-152.

Humphrey, G., Goodale, M., Jakobson, L., \& Servos, P. (1994). The role of surface information in object recognition: Studies of a visual form of agnosic and normal subjects. Perception, 23, 225-238.

JACKSON, S. R., JACKsON, G. M., \& Rosicky, J. (1995). Are non-relevant objects represented in working memory? The effect of non-target objects on reach and grasp kinematics. Experimental Brain Research, 102, 519-530.

JAKobson, L. S., \& Goodale, M. A. (1992). Factors affecting higherorder movement planning: A kinematic analysis of human prehension. Experimental Brain Research, 86, 199-208.

JEANNEROD, M. (1981). Intersegmental coordination during reaching at natural visual objects. In J. Long \& A. Baddeley (Eds.), Attention and performance IX (pp. 153-169). Hillsdale, NJ: Erlbaum.

JonidES, J., \& YANTIS, S. (1988). Uniqueness of abrupt visual onset in capturing attention. Perception \& Psychophysics, 43, 346-354.

Klatzky, R. L., McCloskey, B., Doherty, S., Pellegrino, J., \& Sмiтh, T. (1987). Knowledge about hand shaping and knowledge about objects. Journal of Motor Behaviour, 19, 187-213.

Kritikos, A., Bennett, K. M. B., Dunai, J., \& Castiello, U. (2000). Interference from distracters in reach to grasp movements. Quarterly Journal of Experimental Psychology, 53A, 131-151.

Lhermitte, F. (1983). "Utilization behavior" and its relation to lesions of the frontal lobes. Brain, 106, 237-255.

Mackenzie, C. L., Marteniuk, R. G., Dugas, C., Liske, D., \& EickMEIER, B. (1987). Three-dimensional movement trajectories in a Fitts' task: Implications for control. Quarterly Journal of Experimental Psychology, 39A, 629-647.

Marteniuk, R. G., Leavitt, J. L., Mackenzie, C. L., \& Athenes, S. (1990). Functional relationships between the grasp and transport components in a prehension task. Human Movement Science, 9, 149176.

Marteniuk, R. G., Mackenzie, C. L., Jeannerod, M., Athenes, S., \& Dugas, C. (1987). Constraints on human arm movement trajectories. Canadian Journal of Psychology, 41, 365-378. 
Milner, A., \& Goodale, M. (1995). The visual brain in action. New York: Oxford University Press.

OlDFIELD, R. C. (1971). The assessment and analysis of handedness: The Edinburgh inventory. Neuropsychologia, 9, 97-113.

Remington, R. W., Johnston, J. C., \& Yantis, S. (1992). Involuntary attentional capture by sudden abrupt onsets. Perception \& Psychophysics, 51, 279-290.

Rizzolatti, G., Camarda, R., Fogassi, L., Gentilucci, M., LupPINO, G., \& MATELLI, M. (1988). Functional organization of inferior area 6 in the macaque monkey: II. Area F5 and the control of distal movements. Experimental Brain Research, 71, 491-507.

Robertson, I., Nico, D., \& Hood, B. (1995). The intention to act improves unilateral neglect: Two demonstrations. NeuroReport, 7 , 246-248.

Robertson, I., Nico, D., \& Hood, B. (1997). Believing what you feel: Using proprioceptive feedback to reduce unilateral neglect. Neuropsychology, 11, 53-58.

SAKATA, H., \& TAIRA, M. (1994). Parietal control of hand action. Current Opinion in Neurobiology, 4, 847-856.

Sekuler, A. B., \& Palmer, S. E. (1992). Perception of partly occluded objects: A microgenetic analysis. Journal of Experimental Psychology: General, 121, 95-111.

SivaK, B., \& MACKENZIE, C. L. (1990). Integration of visual information and motor output in reaching and grasping: The contributions of peripheral and central vision. Neuropsychologia, 28, 1095-1116.

Tipper, S. P., Howard, L. A., \& Houghton, G. (2000). Behavioural consequences of selection from neural population codes. In S. Monsell \& J. Driver (Eds.), Attention and performance XVIII: Control of cognitive processes (pp. 223-245). Cambridge, MA: MIT Press.

Tipper, S. P., Howard, L. A., \& JACKSON, S. R. (1997). Selective reaching to grasp: Evidence for distractor interference effects. Visual Cognition, 4, 1-38.

Tipper, S. P., Weaver, B., \& Houghton, G. (1994). Behavioural goals determine inhibitory mechanisms of selective attention. Quarterly Journal of Experimental Psychology, 47A, 809-840.

Vecera, S. P., \& Farah, M. J. (1994). Does visual attention select objects or locations? Journal of Experimental Psychology: General, 123, 146-160.

YANTIS, S., \& Jonides, J. (1984). Abrupt visual onsets and selective attention: Evidence from visual search. Journal of Experimental Psychology: Human Perception \& Performance, 10, 601-621.

YANTIS, S., \& JoNidES, J. (1996). Attentional capture by abrupt onsets: New perceptual objects or visual masking? Journal of Experimental Psychology: Human Perception \& Performance, 22, 1505-1513.

\section{NOTE}

1. I thank H. A. Sedgwick for suggesting this alternative hypothesis.

(Manuscript received May 22, 2000;

revision accepted for publication November 3, 2000.) 